\title{
All-Fiber High-Energy Yb-doped Fiber Amplifier
}

\author{
B. Oktem ${ }^{1}$, H. Kalaycıoğlu ${ }^{2}$, F. Ö. Ilday ${ }^{2}$ \\ ${ }^{I}$ Material Science and Nanotechnology Graduate Program, Bilkent University, 06800 Ankara, Turkey \\ ${ }^{2}$ Department of Physics, Bilkent University, 06800 Ankara, Turkey
}

\begin{abstract}
We report a robust, all-fiber amplifier seeded by a fiber oscillator. Seed pulses at $1 \mathrm{MHz}$ repetition rate are amplified up to $3 \mu \mathrm{J}$, delivering $1 \mu \mathrm{J}$-energy, 170 fs-long pulses. Duration reduces to 120 fs at $1 \mu \mathrm{J}$ amplifier output. These are the highest peak powers from an integrated fiber source.
\end{abstract}

Fiber lasers offer great promise as robust, compact and low-cost sources of powerful ultrafast pulses. In recent years, rapid progress is being reported in this area, with these sources clearly offering the potential for use in applications outside a research laboratory. High-energy fiber amplifiers demonstrated to date are mostly systems employing discrete optical components for coupling of the pump and signal beam and often, a bulk solid-state oscillator to seed the amplifier [1-3]. This counters some of the main advantages of fiber. Recently, we demonstrated a $10-\mathrm{W}$ fiber amplifier at $40 \mathrm{MHz}$ repetition rate, where the entire pulse propagation from fiber seed oscillator is in fiber up to the grating compressor at the end [4]. This architecture with no components requiring alignment increases the robustness of the system significantly. Other groups are reporting lasers incorporating a similar architecture [5].

Here, we demonstrate an all-fiber amplifier, seeded by a fiber oscillator, operating at $1 \mathrm{MHz}$ repetition rate and generating up to $3 \mu \mathrm{J}$ pulse energy, which is compressible to 170 fs. This is to our knowledge the highest peak powers obtained from an integrated fiber amplifier. The pulse energy is limited by the onset of nonlinear effects.

The experimental arrangement of the Yb-doped fiber laser system is shown in Fig. 1. The seed oscillator is a stretchedpulse $\mathrm{Yb}$-doped fiber laser connected to a fiber pulse-stretcher followed by a preamplifier and a high-power amplifier. The amplified pulses are compressed in a grating compressor. The pump light for the entire system is delivered through fiber couplers.

The oscillator has a net group-velocity dispersion (GVD) of $+6150 \mathrm{fs}^{2}$ and produces chirped 2.8 ps-long pulses at $27.7 \mathrm{MHz}$ of repetition rate. An output power of $105 \mathrm{~mW}$ is coupled directly to the amplifier via a $50 \%$ fiber coupler. The fiber stretcher is $50 \mathrm{~m}$-long HI-1060 fiber, stretching the pulses to $62 \mathrm{ps}$. The fiber stretched is followed by a fiber-coupled acousto-optic modulator (AOM) for reducing the repetition rate to $1 \mathrm{MHz}$. The preamplifier amplifies the output of the AOM to $170 \mathrm{~mW}$ of average power (pulse energy of $170 \mathrm{~nJ}$, peak power of $2.75 \mathrm{~kW}$ ). The FWHM values of the spectra were measured to be about $54 \mathrm{~nm}$ and $37 \mathrm{~nm}$ from the $50 \%$ port of the oscillator and the $1 \%$ port of the pre-amplifier, respectively [Fig. 2(a) and (b)].
The power amplifier is pumped co-directionally to protect the pump diodes from optical damage due to leakage of the high-peak-power signal to the pump ports. Pump delivery is accomplished with a multi-port multi-mode pump-signal combiner (MPC) incorporating a single-mode signal feedthrough. 975-nm diodes with $10 \mathrm{~W}$ of maximum power coupled to $105 \mu \mathrm{m}$-diameter fiber are used as pump sources for the power amplifier. The amplifier consists of $2 \mathrm{~m}$-long large mode area double clad fiber with a $20-\mu \mathrm{m}$-core diameter, 0.07 NA ytterbium-doped core and a $125-\mu \mathrm{m}$ octagonal-shaped cladding with NA of 0.46 , spliced to the combined port of the MPC. The output is taken through a fiber isolator-collimator device with a transmission ratio of $90 \%$. This system can generate much higher average output power, so the output is limited by the nonlinear distortion of the pulses, not the available pump power. Higher pulse energies with additional prechirping are possible but residual higher-order dispersion leads to longer pulses.

At an output energy of $1.0 \mu \mathrm{J}$ and $2.95 \mu \mathrm{J}$, the FWHM values of the spectra are measured to be about $27 \mathrm{~nm}$ and 107 $\mathrm{nm}$, respectively [Fig. 2(c) and (d)]. The strong broadening of the pulses at $3 \mu \mathrm{J}$ is attributed to the Raman Effect, which severely limits the compressibility of the pulses: The pulses are compressed to $120 \mathrm{fs}$ and $170 \mathrm{fs}$ (assuming Gaussian shape), respectively [Fig. 3]. The efficiency of the present compressor is $34 \%$ resulting in an average power of $1 \mathrm{~W}$ after compression, corresponding to a pulse energy of $1 \mu \mathrm{J}$ and a pulse peak power of 5.9 MW at a repetition rate of $1 \mathrm{MHz}$. Higher peak powers are possible with the use higher-quality gratings. The laser output is checked against multiple-pulsing and broad pedestal formation by way of long-range autocorrelation, $10 \mathrm{GHz}-$ bandwidth RF spectral measurements and high-resolution optical spectral measurements. The peak power of the pulses is further tested with two-photon absorption measurements. In addition, the laser is used for material processing experiments in our laboratory. Fig. 4 shows a scanning electron microscope (SEM) image of Ti surface processed with this laser. The subwavelength grooves formed within the processed region is interesting from a laser material processing point of view and under investigation.

In conclusion, we have demonstrated a fiber amplifier with no free space beam pump or signal beam propagation, producing 62-ps chirped pulses with $1-3 \mu \mathrm{J}$ pulse energies, which are compressible to $120 \mathrm{fs}$ and $170 \mathrm{fs}$, respectively, via a grating compressor. The laser system is extremely robust and is currently being used for material processing applications in our laboratory. 


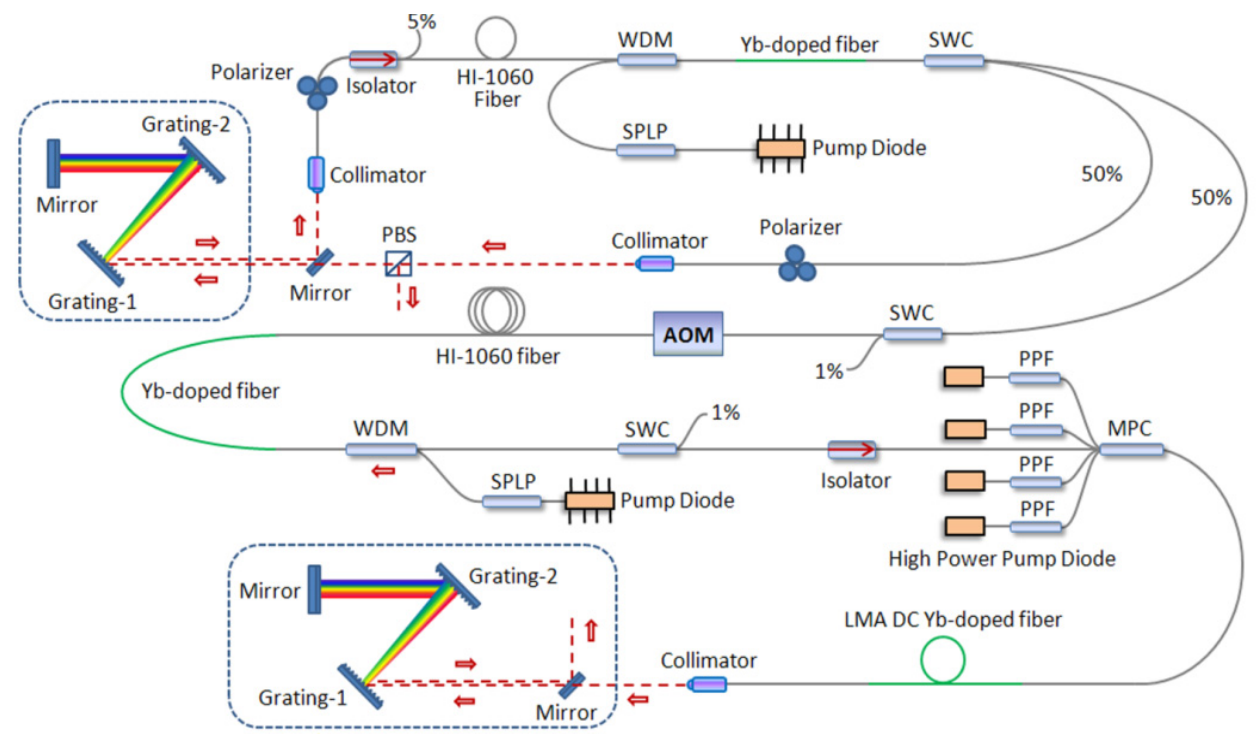

Fig. 1. Schematic diagram of the high-energy femtosecond Yb-doped fiber laser, PBS: polarizing beam splitter, WDM: wavelength-division multiplexer, SWC: single window coupler, LMA: large mode area, SPLP: single mode pump laser protector, PPF: pump protection filter, MPC: multi-mode pump-signal combiner.
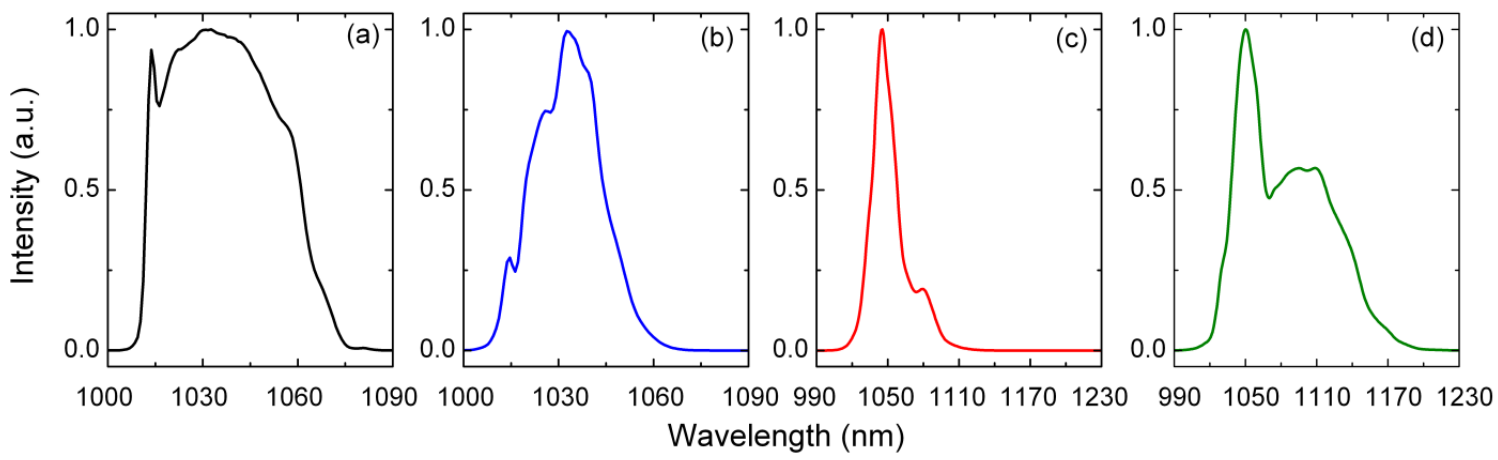

Fig. 2. Measured spectra of the pulse from (a) the fiber port of the oscillator, (b) $1 \%$ fiber port of the pre-amplifier, (c) the power amplifier at $1 \mathrm{~W}$ of average power, (d) the power amplifier at $2.95 \mathrm{~W}$ of average power.

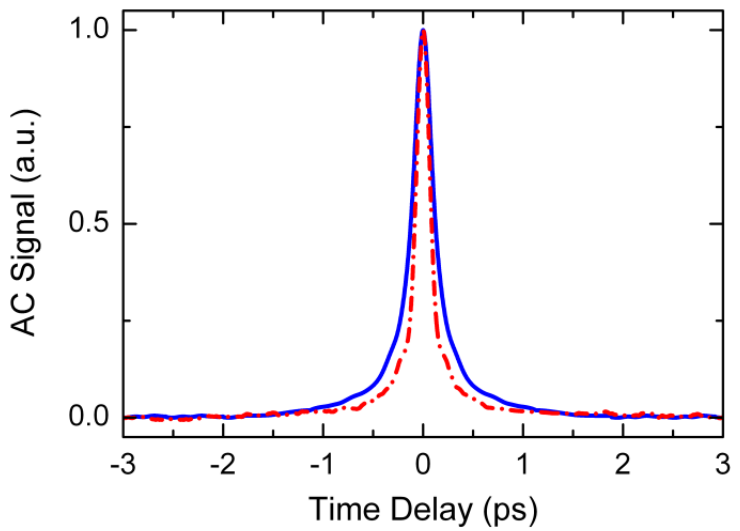

Fig. 3. Intensity autocorrelation of the pulse from the grating compressor of the high power amplifier at $1 \mathrm{~W}$ of power (red dash-dotted curve) and at 2.95 $\mathrm{W}$ of power (blue solid curve).

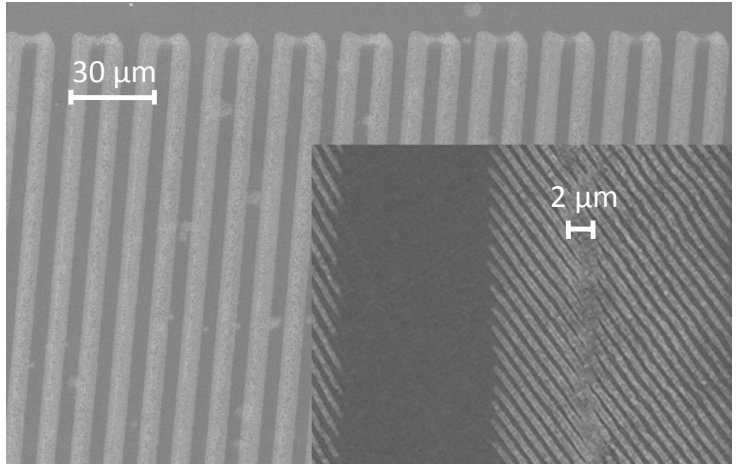

Fig. 4. SEM micrographs of laser patterned titanium surface with sub-wavelength groove structures within the laser-modified areas.

\section{References}

[1] J. Limpert, T. Schreiber, T. Clausnitzer, K. Zöllner, H. Fuchs, E. Kley, H. Zellmer, and A. Tünnermann, Optics Express, Vol. 10, pp. 628-638, 2002.

[2] S. Hädrich, J. Rothhardt, T. Eidam, J. Limpert, and A. Tünnermann, Optics Express, Vol. 17, Issue 5, pp. 3913-3922, 2009.

[3] Y. Zaouter, J. Boullet, E. Mottay, and E. Cormier, Optics Letters, Vol. 33, Issue 13, pp. 1527-1529, 2008.

[4] P. K. Mukhopadhyay, K. Özgören, İ. L. Budunoğlu, and F. Ö. İlday, IEEE J. Sel. Top. Quant.,vol. 15, pp. 145, 2009.

[5] S. Zhou, T. Takamido, R. Bhandari, A. Chong and Frank Wise, Proc. SPIE, Vol. 7195, pp. 719516, 2009. 